\title{
STRONG DITKIN ALGEBRAS WITHOUT BOUNDED RELATIVE UNITS
}

\author{
J. F. FEINSTEIN
}

(Received 12 November 1998)

\begin{abstract}
In a previous note the author gave an example of a strong Ditkin algebra which does not have bounded relative units in the sense of Dales. In this note we investigate a certain family of Banach function algebras on the one point compactification of $\mathbb{N}$, and see that within this family are many easier examples of strong Ditkin algebras without bounded relative units in the sense of Dales.
\end{abstract}

Keywords and phrases. Banach function algebras, functional analysis, strong Ditkin algebras, bounded relative units.

1991 Mathematics Subject Classification. 46J10.

1. Introduction. Regularity conditions for Banach function algebras have important applications in several areas of functional analysis, including automatic continuity theory and the theory of Wedderburn decompositions (see, for example, [2]). There is also a close connection between regularity and the theory of decomposable operators, as was shown by Neumann in [6]. It is thus important both to investigate the connections between these regularity conditions and other conditions that a Banach function algebra may satisfy, and also to find out what the relationships are between these regularity conditions. For a survey of many regularity conditions and their relationships to each other, see [5].

The aim of this note is to investigate some of the stronger regularity conditions for a particular class of Banach function algebras, following on from the work in [4]. In [4], the author solved a problem posed by Bade (see [3]), by showing that every strong Ditkin algebra has bounded relative units at each point of its character space. The proof was very short and elementary. In fact, unknown to the author, essentially the same argument had been used earlier by Bachelis, Parker and Ross [1] to prove a special case of the same result. (I am grateful to Jan Stegeman for later pointing out the existence of this paper to me.) Also in [4], the author constructed an example of a strong Ditkin algebra which does not satisfy the stronger condition of having bounded relative units in the sense of Dales (see below). In Section 2 we shall introduce a particular class of Banach function algebras on $\mathbb{N}_{\infty}$, and determine precisely which combinations of regularity properties are possible for algebras in this class. In particular we shall show that this class includes many further examples of strong Ditkin algebras which do not have bounded relative units in the sense of Dales. These examples are easier than the example constructed in [4], and show that interesting combinations of properties are possible even when working with Banach function 
algebras on $\mathbb{N}_{\infty}$. First, we recall the standard notation and definitions which we shall need.

NotATion. For any compact, Hausdorff space $X$ we denote the algebra of all continuous, complex-valued functions on $X$ by $C(X)$. Let $A$ be a commutative, unital algebra. We denote by $\Phi_{A}$ the character space of $A$. Let $S, T$ be subsets of $A$. We denote by $S \cdot T$ the set

$$
\{a b: a \in S, b \in T\} .
$$

We denote the one point compactification of $\mathbb{N}$ by $\mathbb{N}_{\infty}$ (so that $\mathbb{N}_{\infty}=\mathbb{N} \cup\{\infty\}$ ). For $f \in C\left(\mathbb{N}_{\infty}\right),\|f\|_{\infty}$ denotes the usual uniform norm of $f$ on $\mathbb{N}_{\infty}$, so that

$$
\|f\|_{\infty}=\sup \{|f(n)|: n \in \mathbb{N}\} .
$$

DEFINITION 1.1. Let $X$ be a compact space. A function algebra on $X$ is a subalgebra of $C(X)$ which contains all of the constant functions and which separates the points of $X$. A Banach function algebra on $X$ is a function algebra on $X$ with a complete algebra norm.

Throughout we shall consider only unital Banach algebras. Using the Gelfand transform, every commutative, semisimple Banach algebra may be regarded as a Banach function algebra on its character space.

NotATion. Let $A$ be a (unital) Banach function algebra on $\Phi_{A}$ and let $E$ be a closed subset of $\Phi_{A}$. We define two ideals $J(E), I(E)$ in $A$ as follows

$$
\begin{gathered}
J(E)=\{f \in A: f \text { vanishes on some neighbourhood of } E\} ; \\
I(E)=\{f \in A: f(E) \subseteq\{0\}\} .
\end{gathered}
$$

For $\phi \in \Phi_{A}$, we set $M_{\phi}=I(\{\phi\})$ and we denote $J(\{\phi\})$ by $J_{\phi}$.

DeFinition 1.2. Let $A$ be a Banach function algebra on $\Phi_{A}$ and let $\phi \in \Phi_{A}$. Then $A$ is strongly regular at $\phi$ if $J_{\phi}$ is dense in $M_{\phi}$; A satisfies Ditkin's condition at $\phi$ if $J_{\phi} \cdot M_{\phi}$ is dense in $M_{\phi}$. We say that $A$ has bounded relative units at $\phi$ if there exists $C \geq 1$ such that, for all compact sets $E \subseteq \Phi_{A} \backslash\{\phi\}$, there is $f \in J_{\phi}$ with $\|f\| \leq C$ such that $f(E) \subseteq\{1\}$. The algebra $A$ is strongly regular if it is strongly regular at every point of $\Phi_{A} ; A$ is a Ditkin algebra if it satisfies Ditkin's condition at every point of $\Phi_{A} ; A$ is a strong Ditkin algebra if every maximal ideal of $A$ has a bounded approximate identity and $A$ is strongly regular. The Banach function algebra $A$ has spectral synthesis if, for every closed set $E \subseteq \Phi_{A}, J(E)$ is dense in $M(E)$.

Clearly every Ditkin algebra is strongly regular. In the special case where $\Phi_{A}=\mathbb{N}_{\infty}$ it is easy to see that if $A$ is a Ditkin algebra, then $A$ has spectral synthesis (this is not true in general).

There are two definitions for a Banach function algebra $A$ to have bounded relative units. One is that, for every $\phi \in \Phi_{A}, A$ has bounded relative units at $\phi$. If this condition 
holds we shall say that $A$ has bounded relative units in the sense of Bade. The other definition, used more frequently, is stronger, insisting that the constant $C$ involved does not depend on $\phi$. If this stronger condition holds we shall say that $A$ has bounded relative units in the sense of Dales. For uniform algebras, these two conditions are equivalent. But they differ for general Banach function algebras, as was shown in [4].

The following result combines some standard theory with [4, Thm. 5].

Proposition 1.3. Let $A$ be a Banach function algebra. Then $A$ is a strong Ditkin algebra if and only if $A$ is strongly regular and has bounded relative units in the sense of Bade.

As we mentioned earlier, in [4] there is an example of a strong Ditkin algebra which does not have bounded relative units in the sense of Dales. In the next section we shall give some easier examples of this.

2. Banach function algebras on $\mathbb{N}_{\infty}$. We shall now introduce the class of Banach function algebras that we shall work with. From now on we shall use $\alpha$ to denote a sequence of positive real numbers, with $\alpha=\left(\alpha_{n}\right)_{n=1}^{\infty}$. Given such a sequence $\alpha$, we define $A_{\alpha}$ by

$$
A_{\alpha}=\left\{f \in C\left(\mathbb{N}_{\infty}\right): \sum_{n=1}^{\infty} \alpha_{n}|f(n+1)-f(n)|<\infty\right\} .
$$

It is easy to see that $A_{\alpha}$ is a subalgebra of $C\left(\mathbb{N}_{\infty}\right)$, and that $A_{\alpha}$ is a Banach function algebra, where the norm of a function $f \in A_{\alpha}$ is given by

$$
\|f\|=\|f\|_{\infty}+\sum_{n=1}^{\infty} \alpha_{n}|f(n+1)-f(n)| .
$$

It is also easy to see that the character space of $A_{\alpha}$ is just $\mathbb{N}_{\infty}$ (this follows from [4, Prop. 6], for example).

We shall now investigate the further properties of these algebras. We shall see that $A_{\alpha}$ is always a Ditkin algebra (and hence strongly regular). In fact we will see that $A_{\alpha}$ has a stronger property: for all $x \in \mathbb{N}_{\infty}, M_{x}$ has an approximate identity (not necessarily bounded) consisting of a sequence of elements of $J_{x}$. Of course, for each $x \in \mathbb{N}$, it is clear that $J_{x}=M_{x}$, and that these ideals have an identity. So we only need to check what happens at the point $\infty$. We begin by looking at the particular sequence of functions $\left(e_{k}\right) \subseteq A_{\alpha}$ defined by

$$
e_{k}(n)= \begin{cases}1, & \text { if } n \leq k \\ 0, & \text { otherwise }\end{cases}
$$

We shall see that this sequence of functions in $J_{\infty}$ always has a subsequence which is an approximate identity for $M_{\infty}$, although the sequence $\left(e_{k}\right)$ need not itself be such an approximate identity. First we need an elementary lemma.

LEMMA 2.1. Let $\left(n_{k}\right)_{k=1}^{\infty}$ be a strictly increasing sequence of natural numbers. Then the following conditions are equivalent 
(a) $\left(e_{n_{k}}\right)$ is an approximate identity for $M_{\infty}$;

(b) for all $f \in M_{\infty}, \lim _{k \rightarrow \infty} \alpha_{n_{k}} f\left(n_{k}+1\right)=0$;

(c) for all $f \in M_{\infty}, \lim _{k \rightarrow \infty} \alpha_{n_{k}} f\left(n_{k}\right)=0$.

Proof. For any $f \in A_{\alpha}$ we know that $\sum_{k=1}^{\infty} \alpha_{n}|f(n+1)-f(n)|<\infty$, and so $\lim _{n \rightarrow \infty} \alpha_{n}|f(n+1)-f(n)|=0$. From this it is clear that (b) and (c) are equivalent. Now let $f \in M_{\infty}$. Then

$$
\left(f-e_{n_{k}} f\right)(j)= \begin{cases}0, & \text { if } j \leq n_{k} \\ f(j), & \text { otherwise }\end{cases}
$$

We have that

$$
\left\|f-e_{n_{k}} f\right\|=\left\|f-e_{n_{k}} f\right\|_{\infty}+\sum_{j=n_{k}+1}^{\infty}\left(\alpha_{j}|f(j+1)-f(j)|\right)+\alpha_{n_{k}}\left|f\left(n_{k}+1\right)\right| .
$$

Since the first two terms on the right hand side of (2.1) tend to zero as $k \rightarrow \infty$, it is clear that $\lim _{k \rightarrow \infty}\left\|f-e_{n_{k}} f\right\|=0$ if and only if $\lim _{k \rightarrow \infty} \alpha_{n_{k}} f\left(n_{k}+1\right)=0$. It is now immediate that (a) and (b) are equivalent, as required.

The sequence $\left(e_{k}\right)$ is not always an approximate identity for $M_{\infty}$. For example, suppose that $\alpha$ is the sequence $\left(\alpha_{n}\right)$ defined by

$$
\alpha_{n}= \begin{cases}1, & \text { if } n \text { is odd, } \\ \frac{n}{2}, & \text { if } n \text { is even. }\end{cases}
$$

Then $\left(e_{k}\right)$ is not an approximate identity for $M_{\infty}$. This can be seen by considering the function $f \in C\left(\mathbb{N}_{\infty}\right)$ which satisfies

$$
f(j)=2^{-k},
$$

whenever $j, k \in \mathbb{N}$ are such that $2^{k-1} \leq j<2^{k}$.

When $j=2^{k}-1, \alpha_{j}|f(j+1)-f(j)|=2^{-k-1}$. All other $|f(j+1)-f(j)|$ are 0 , so that $f \in A_{\alpha}$, and $f \in M_{\infty}$. But $\alpha_{2^{k}} f\left(2^{k}\right)=1 / 4$ for all $k$, and so $\alpha_{n} f(n)$ does not tend to 0 as $n \rightarrow \infty$. Thus, by Lemma 2.1, $\left(e_{k}\right)$ is not an approximate identity for $M_{\infty}$.

We now continue our investigation into when $\left(e_{n_{k}}\right)$ is an approximate identity.

THEOREM 2.2. Let $\left(n_{k}\right)$ be as in Lemma 2.1

(a) Suppose that, for all $k \in \mathbb{N}, \alpha_{n_{k}}=\inf \left\{\alpha_{j}: j \geq n_{k}\right\}$. Then $\left(e_{n_{k}}\right)$ is an approximate identity for $M_{\infty}$.

(b) The sequence $\left(e_{n_{k}}\right)$ is a bounded approximate identity for $M_{\infty}$ if and only if the sequence $\left(\alpha_{n_{k}}\right)$ is bounded.

Proof. To prove (a), suppose that $\left(\alpha_{n_{k}}\right)$ satisfies the given condition. Let $f \in M_{\infty}$. Then we have, for $k \in \mathbb{N}$,

$$
f\left(n_{k}\right)=\sum_{j=n_{k}}^{\infty}(f(j)-f(j+1)) .
$$


Thus

$$
\begin{aligned}
\left|\alpha_{n_{k}} f\left(n_{k}\right)\right| & \leq \sum_{j=n_{k}}^{\infty} \alpha_{n_{k}}|f(j)-f(j+1)| \\
& \leq \sum_{j=n_{k}}^{\infty} \alpha_{j}|f(j)-f(j+1)| .
\end{aligned}
$$

Since the last sum tends to 0 as $k \rightarrow \infty$, it follows that $\lim _{k \rightarrow \infty} \alpha_{n_{k}} f\left(n_{k}\right)=0$. It now follows from Lemma 2.1 that $\left(e_{n_{k}}\right)$ is an approximate identity for $M_{\infty}$.

To prove (b), first note that, for all $k$,

$$
\left\|e_{n_{k}}\right\|=1+\alpha_{n_{k}}
$$

Thus it is clear that the sequence $\left(e_{n_{k}}\right)$ is bounded in norm if and only if $\left(\alpha_{n_{k}}\right)$ is bounded. It remains to show that, in this case, $\left(e_{n_{k}}\right)$ is also an approximate identity for $M_{\infty}$. But this is immediate from Lemma 2.1. The result follows.

We can now see that $\left(e_{k}\right)$ is itself often an approximate identity.

COROLlaRY 2.3. If the sequence $\alpha$ is either nondecreasing or bounded then $\left(e_{k}\right)$ is an approximate identity for $M_{\infty}$.

Proof. This is immediate from Theorem 2.2.

We can now prove our main result about the existence of approximate identities in $M_{\infty}$.

THEOREM 2.4. The sequence $\left(e_{k}\right)$ always has a subsequence which is an approximate identity in $M_{\infty}$ in $A_{\alpha}$.

Proof. If $\left(\alpha_{n}\right)$ has a bounded subsequence, then the result is immediate from Theorem 2.2(b). Thus we may assume that $\left(\alpha_{n}\right)$ diverges to $\infty$. But then it is easy to choose $\left(n_{k}\right)$ such that the conditions of Theorem 2.2(a) are satisfied, and so $\left(e_{n_{k}}\right)$ is an approximate identity for $M_{\infty}$.

As a corollary, we obtain immediately the fact that each $A_{\alpha}$ is a Ditkin algebra.

COROLLARY 2.5. For every sequence of positive real numbers $\alpha$, the Banach function algebra $A_{\alpha}$ is a Ditkin algebra.

Proof. For $x \in \mathbb{N}$ it is clear that $J_{x}=M_{x}$ and that these ideals have an identity. It remains to check that $J_{\infty} \cdot M_{\infty}$ is dense in $M_{\infty}$, but this is immediate from Theorem 2.4. The result follows.

Of course this means that $A_{\alpha}$ is always strongly regular. By the nature of the algebra, it follows that $A_{\alpha}$ is always separable. Since $\Phi_{A_{\alpha}}=\mathbb{N}_{\infty}$, it also follows that $A_{\alpha}$ has spectral synthesis.

We now characterise those sequences $\alpha$ for which the algebra $A_{\alpha}$ has various regularity properties. 
THEOREM 2.6. Let $\alpha=\left(\alpha_{n}\right)_{n=1}^{\infty}$ be any sequence of positive real numbers. Then

(a) $A_{\alpha}$ is a strong Ditkin algebra if and only if $\liminf _{n \rightarrow \infty} \alpha_{n}<\infty$, i.e., $\left(\alpha_{n}\right)$ does not diverge to $\infty$.

(b) In $A_{\alpha}, M_{\infty}$ has a bounded approximate identity if and only if $\liminf _{n \rightarrow \infty} \alpha_{n}<\infty$.

(c) The Banach function algebra $A_{\alpha}$ has bounded relative units in the sense of Bade if and only if $\liminf _{n \rightarrow \infty} \alpha_{n}<\infty$.

(d) The algebra $A$ has bounded relative units in the sense of Dales if and only if the sequence $\left(\alpha_{n}\right)$ is bounded.

Proof. We know that $A_{\alpha}$ is a Ditkin algebra (and hence strongly regular). Also, for all $x \in \mathbb{N}, J_{x}=M_{x}$, and $M_{x}$ has an identity. Thus, by Proposition 1.3, it is clear that (a), (b), (c) are equivalent, and that for each of these we only need to check the relevant condition at the point $\infty$. Suppose first that $\liminf _{n \rightarrow \infty} \alpha_{n}<\infty$. Then $\left(\alpha_{n}\right)$ has a bounded subsequence, and so it follows from Theorem 2.2(b) that $M_{\infty}$ has a bounded approximate identity. Thus $A_{\alpha}$ is a strong Ditkin algebra, and has bounded relative units in the sense of Bade.

Conversely, suppose that $A_{\alpha}$ has bounded relative units in the sense of Bade. Then there is a norm bounded sequence of functions $\left(f_{n}\right) \subseteq J_{\infty}$ such that, for all $n \in N$, $f_{n}(\{n\})=\{1\}$. Set $M=\sup _{n}\left\|f_{n}\right\|$. We show that $\liminf _{n \rightarrow \infty} \alpha_{n} \leq M$. Suppose, for contradiction, that $\liminf _{n \rightarrow \infty} \alpha_{n}>M$. Choose $N \in \mathbb{N} \operatorname{such}$ that $\inf \left\{\alpha_{j}: j \geq N\right\}>M$. Then we have

$$
\begin{aligned}
1 & =\left|f_{N}(N)\right| \\
& =\left|\sum_{j=N}^{\infty}\left(f_{N}(j)-f_{N}(j+1)\right)\right| \\
& \leq \sum_{j=N}^{\infty}\left|\left(f_{N}(j)-f_{N}(j+1)\right)\right| \\
& \leq \frac{\left\|f_{N}\right\|}{\inf \left\{\alpha_{j}: j \geq N\right\}}<1 .
\end{aligned}
$$

This contradiction proves that, as claimed, $\liminf _{n \rightarrow \infty} \alpha_{n} \leq M<\infty$. Parts (a) to (c) now follow.

To see (d), suppose first that $\left(\alpha_{n}\right)$ is bounded. Set $M=\sup _{n} \alpha_{n}$. For $n \in \mathbb{N}$, the norm of the identity of $M_{n}$ is at most $2 M+1$. Also, with $e_{n}$ as above, $\left\|e_{n}\right\| \leq M+1$, and so $A_{\alpha}$ has bounded relative units in the sense of Dales, with bound $2 M+1$. Conversely, since the norm of the identity in $M_{n}$ is at least $\alpha_{n}$, it is immediate that if $A_{\alpha}$ has bounded relative units in the sense of Dales then $\left(\alpha_{n}\right)$ must be bounded. The result follows.

This result can be used to give many elementary examples of strong Ditkin algebras which do not have bounded relative units in the sense of Dales.

COROLLARY 2.7. Let $\alpha$ be any sequence of positive real numbers such that $\alpha$ is unbounded but does not diverge to $\infty$. Then $A_{\alpha}$ is a strong Ditkin algebra which does not have bounded relative units in the sense of Dales.

Proof. This is immediate from Theorem 2.6. 


\section{REFERENCES}

[1] G. F. Bachelis, W. A. Parker, and K. A. Ross, Local units in $L^{1}(G)$, Proc. Amer. Math. Soc. 31 (1972), 312-313. MR 44\#5794. Zbl 252.43013.

[2] W. G. Bade and H. G. Dales, The Wedderburn decomposability of some commutative Banach algebras, J. Funct. Anal. 107 (1992), no. 1, 105-121. MR 93d:46090. Zbl 765.46036.

[3] W. G. Bade et al, Open problems, Conference on Automatic Continuity and Banach Algebras (Canberra) (R.-J. Loy, ed.), Proceedings of the Centre for Mathematical Analysis, vol. 21, Australian National University, 1989. MR 90f:46002. Zbl 717.46040.

[4] J. F. Feinstein, A note on strong Ditkin algebras, Bull. Austral. Math. Soc. 52 (1995), no. 1, 25-30. MR 96f:46097. Zbl 836.46041.

[5]__ Regularity conditions for Banach function algebras, Function spaces (Edwardsville, IL, 1994) (New York) (Krzysztof Jarosz, ed.), Lect. Notes Pure Appl. Math., vol. 172, Marcel Dekker, 1995, pp. 117-122. MR 96h:46077. Zbl 838.46042.

[6] M. M. Neumann, Commutative Banach algebras and decomposable operators, Monatsh. Math. 113 (1992), no. 3, 227-243. MR 93e:46056. Zbl 765.47010.

Feinstein: School of Mathematical SCiences, PuRe Mathematics Division, University OF NOTTINGHAM, NOTTINGHAM NG7 2RD, ENGLAND

E-mail address: Joe1. Feinstein@nottingham.ac.uk 


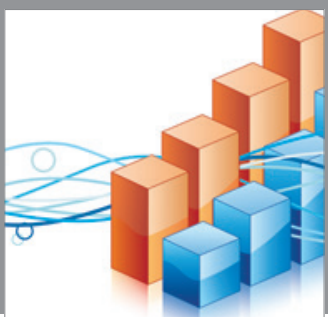

Advances in

Operations Research

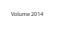

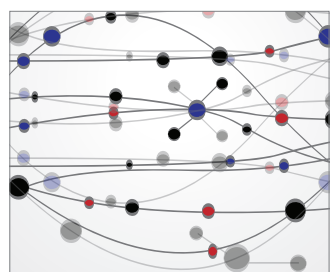

\section{The Scientific} World Journal
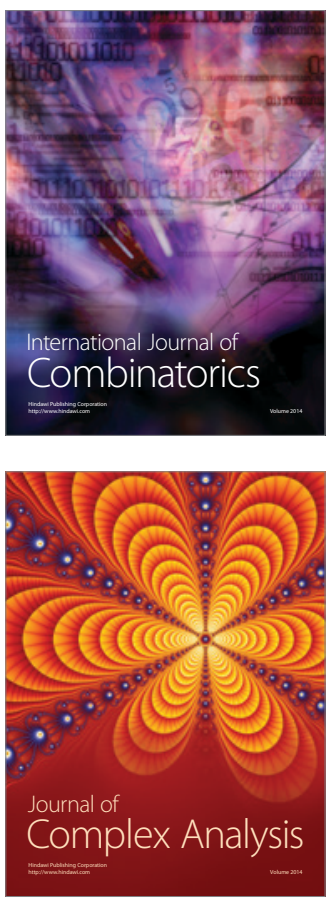

International Journal of

Mathematics and

Mathematical

Sciences
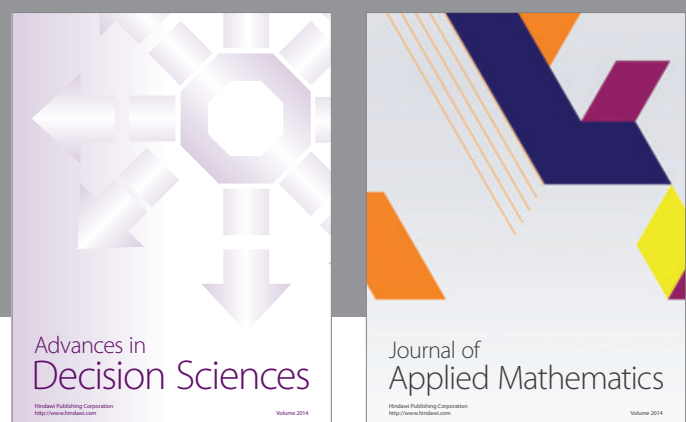

Journal of

Applied Mathematics
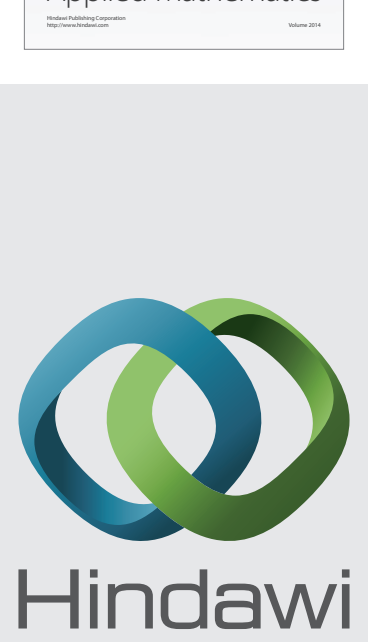

Submit your manuscripts at http://www.hindawi.com
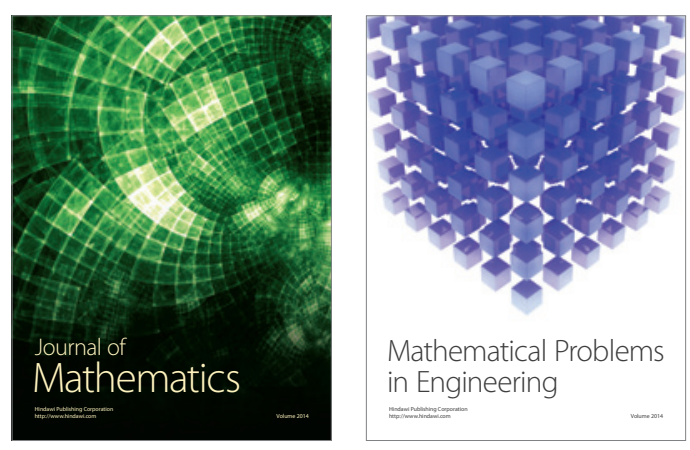

Mathematical Problems in Engineering
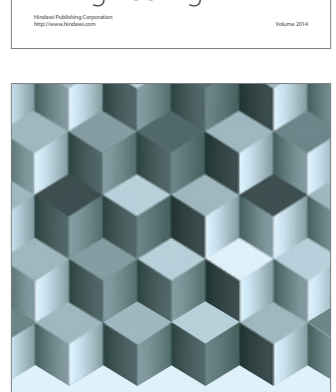

Journal of

Function Spaces
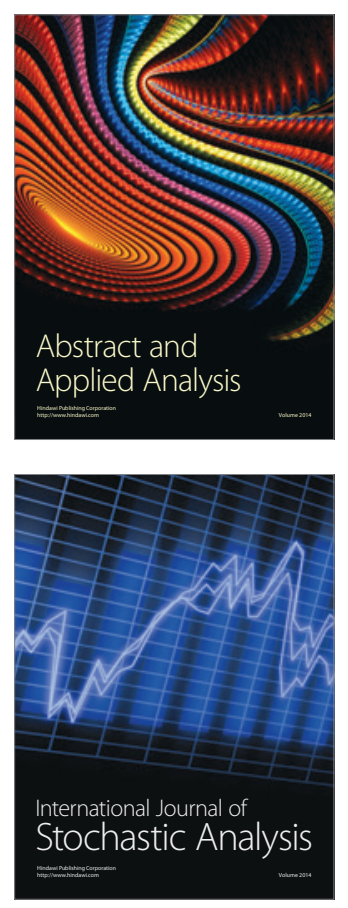

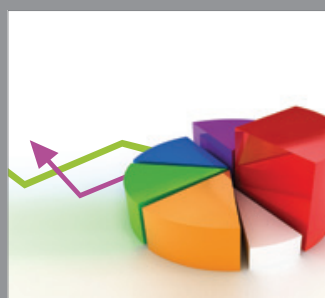

ournal of

Probability and Statistics

Promensencen
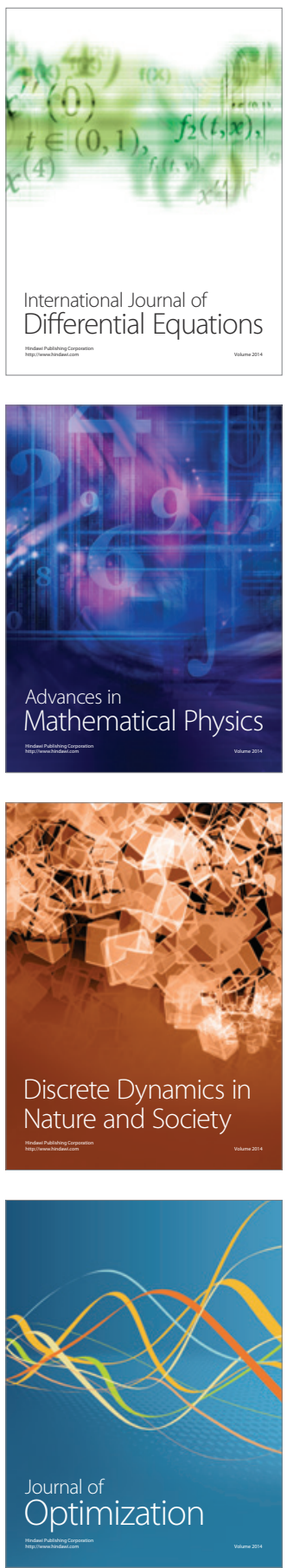